Classification

Physics Abstracts

$21.10 \mathrm{D}-25.85 \mathrm{E}$

\title{
Fission from saddle to exit : influence of curvature and compression energies
}

\author{
J. Treiner $(*)$, R. W. Hasse \\ Institut Laue-Langevin, 156X, 38042 Grenoble Cedex, France
}

and P. Schuck

Institut des Sciences Nucléaires, 38026 Grenoble Cedex, France

(Reçu le 19 avril 1983, révisé le 6 juillet, accepté le 11 juillet 1983)

\begin{abstract}
Résumé. - Nous étudions l'influence des termes qui vont au-delà du modèle standard goutte liquide sur la pente de la vallée de fission. Une réduction importante de la pente est obtenue avec des valeurs de l'énergie de courbure calculées à partir des forces effectives couramment utilisées : nous corroborons ainsi les calculs microscopiques récemment effectués par Berger $e t$ al.
\end{abstract}

\begin{abstract}
We investigate the influence of terms beyond the standard Liquid Drop Model on the slope of the fission valley. An important reduction of the slope is obtained when using values of the curvature energy calculated from effective forces currently in use, thus corroborating a recent microscopic calculation by Berger et al.
\end{abstract}

In a recent paper, Berger, Girod and Gogny, hereafter referred to as BGG [1] have presented a microscopic description of nuclear fission based on a Hartree-Fock-Bogoliubov (HFB) calculation of the potential energy surface $E=V\left(q_{1}, q_{2} \ldots\right)$ of ${ }^{240} \mathrm{Pu}$, where $q_{1}, q_{2} \ldots$ denotes some set of collective variables characterizing the shape of the nucleus. Some important features of this potential energy surface can be seen in figures 1 and 2 of BGG. In figure 2, the descent from saddle to scission is presented as a function of two collective variables, namely the quadrupole moment $Q_{2}$ and the hexadecapole moment $Q_{4}$. Certain aspects of BGG's results are common to other calculations : the fission valley is separated by a ridge from the fusion region, corresponding to fragmented configurations with large and negative potential energies : this ridge vanishes with increasing deformation (both valleys have to join somewhere) at the so-called exit point. However, figure 2 of BGG presents a new and striking feature : the mean slope of the fission valley is much smaller than that currently obtained in the Liquid Drop Model (LDM) : the gain in energy between saddle and exit is of the order of 4-5 MeV in BGG's paper [2] while it is of the order of

$\left(^{*}\right)$ On leave of absence from DPT-IPN, F-91046 Orsay Cedex, France. 

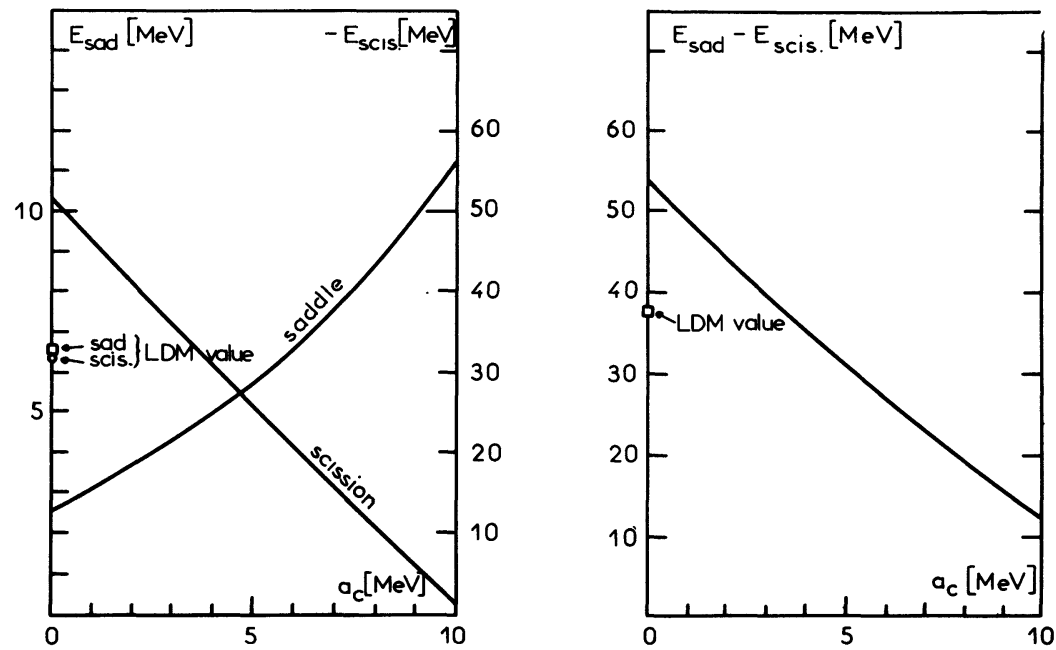

Fig. 1. - Left : plot of saddle and scission energies for ${ }^{240} \mathrm{Pu}$ as functions of the curvature energy coefficients. Right : plot of the difference $E_{\text {saddle }}-E_{\text {scission }}$ as a function of the curvature energy coefficient.
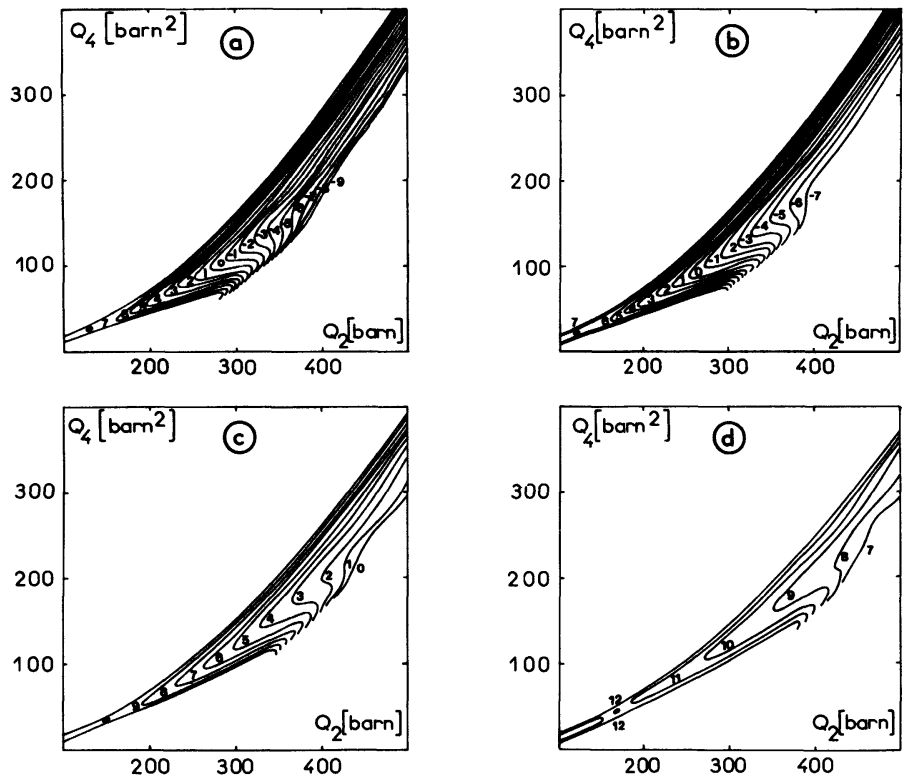

Fig. 2. - Potential energy surfaces for ${ }^{240} \mathrm{Pu}$. $a$ : LDM calculation (no curvature, no compression), $b, c, d$ : correspond to values of the curvature energy coefficient of $a_{\mathrm{c}}=5,8,10 \mathrm{MeV}$, respectively, with inclusion of the compression effect.

15-20 MeV in the LDM (see e.g. Ref. 3). Such a discrepancy will have important consequences on the dynamics of the fission process; in particular « the slow variation of the potential energy surface during (the) evolutive phase justifies the adiabatic assumption on which our interpretation of the scission process is based " [1]. Indeed if the surface is almost flat the whole picture of the fission process adopted in the past (see e.g. Refs. 3,4 ) must be revisited : there would be essentially 
no damping because of no quasi-particle excitation; moreover the fission process would be so slow that tunnelling across the ridge could occur, i.e. before the system reaches the exit point.

It is therefore interesting to locate the origin of the discrepancy in the calculation of the mean slope of the fission valley between the usual LDM and BGG. Two main effects can be investigated (i) the shell and pairing corrections which, in the Strutinsky approach [5], have to be added to the LDM part in order to compare with a microscopic calculation and (ii) the liquid drop part itself, which is truncated in the standard LDM, to surface, surface asymmetry and Coulomb contributions. In fact some other effects could also play a rôle : for example, BGG allow for a possible left-right asymmetry while the LDM obviously does not; this tends to decrease slightly the slope of the fission valley [2] ; also in the LDM calculations, one usually uses a parametrized family of shapes whereas in BGG's paper the shapes come out naturally from a minimization of the energy under constraints.

Among all these possible effects, we do not think that the shell and pairing corrections can be convincing candidates. Strutinsky type calculations of the potential energy surface show that shell effects are important in explaining the local structure of the surface near the saddle point but do not change the general features of the landscape towards exit (see e.g. Ref. 3). To our opinion, the drastic discrepancy in the mean slope of the fission valley should rather be looked at as an indication of a qualitative effect present already in the smooth part of BGG's surface but not in the standard LDM. The simplest effect one can think of is that of curvature and compression energies, which we shall now analyse in details.

The choice usually made for the curvature energy coefficient $a_{\mathrm{c}}$ in the recent macroscopic approaches to nuclear masses is $a_{\mathrm{c}}=0$ (see e.g. Refs. 6, 7; in the folding model of Ref. 7, $a_{\mathrm{c}}=0$ by construction). When starting with a microscopic effective interaction, one expects a positive value of $a_{\mathrm{c}}$ because the defect of binding energy at the surface, which gives rise to the surface energy, is greater for small systems (i.e. large curvature) than for large ones. However an accurate value of $a_{\mathrm{c}}$ cannot be extracted through a fitting procedure to calculated masses, because large error bars and strong correlations to the other coefficients of the mass formula are obviously obtained in such a procedure [8]. Let us now briefly recall how one can derive a simple analytical formula for $a_{\mathrm{c}}$ within the framework of an Energy Density Formalism (EDF).

Such an EDF, in which the energy of the nucleus appears as a functional of the local densities only, can be constructed by using a Density Matrix Expansion of the full one-body density matrix [9] for the potential energy part together with an Extended Thomas Fermi approximation for the kinetic energy part. If one then represents the neutron and proton densities by Fermi type distributions, an expansion of the energy of the nucleus in powers of the ratio of the surface diffuseness to the radius can be obtained using the results of reference 10. By this method simple analytical formulae can be given for the surface energy $a_{\mathrm{s}}$, the surface symmetry energy $a_{\mathrm{ss}}$, the curvature energy $a_{\mathrm{c}}$ and the constant term $a_{0}$ in the mass formula. The expressions are particularly transparent when one uses a simple Fermi distribution for the density (more elaborate parameterizations are considered in Ref. 11). One gets in particular for the curvature energy

$$
a_{\mathrm{c}}=\frac{3 a \sigma}{\rho_{\mathrm{nm}}}+\frac{3}{r_{\mathrm{nm}}^{2}}\left[\frac{\hbar^{2}}{2 m} \beta-\frac{m^{*}}{m} V_{\mathrm{so}} \rho_{\mathrm{nm}}^{2}\right]
$$

where $a$ is the surface diffuseness, $\sigma$ the surface tension, $\rho_{\mathrm{nm}}$ and $r_{\mathrm{nm}}$ the saturation density and the nuclear radius constant, respectively, of infinite nuclear matter, $V_{\text {so }}$ a coefficient related to the strength of the spin-orbit interaction $\left(V_{\text {so }} \simeq-200 \mathrm{MeV} \cdot \mathrm{fm}^{6}\right), \beta$ the coefficient of the Weizsäcker term in the ETF functional and $m^{*}$ the effective mass in nuclear matter. We want to stress the fact that $a_{\mathrm{c}}$ appears as the sum of two terms of the same positive sign, so that it cannot vanish for some particular values of the parameters of the effective interaction giving rise to our EDF. Indeed the numerical values obtained for $a_{\mathrm{c}}$ in reference 11 lie all in the range (10-14) $\mathrm{MeV}$, and from the approximate formula 1 one understands why $a_{\mathrm{c}}$ does not vary much with the effec- 
tive interaction : it is related to physical quantities that are well determined (notice that the dominant term in the r.h.s. of eq. 1 is the first one), so that any realistic effective interaction should reproduce them. Our values of $a_{\mathrm{c}}$ agree with previous calculations, e.g. the Thomas Fermi calculations of reference 12, the ETF calculations of reference 13, and with the estimate given in reference 14 for the Skyrme type interactions SIII and SkM (9-10 MeV), obtained from a fit to calculated masses. Note that the coefficient of the $A^{1 / 3}$-term in the mass formula is not to be identified with the curvature energy; it contains a compression term

$$
-2 \frac{a_{\mathrm{s}}^{2}}{K} A^{1 / 3}
$$

(where $K$ is the incompressibility modulus in infinite nuclear matter) arising from the central compression of the density due to the surface tension. This term, which lies in the range - (2 ...3) $A^{1 / 3} \mathrm{MeV}$ depending on the values of $K$, varies with the square of the surface of the nucleus when it is deformed. It thus plays an important rôle in the calculation of the potential energy surface, as we shall see below.

Concerning the isovector properties, we shall consider only the surface symmetry energy, which has been calculated for Gogny's force [17] in references 11,15 . We shall neglect the curvature symmetry energy, $a_{\mathrm{cs}}$ for which no simple expression has been derived so far. Let us, however, mention that for the whole set of Skyrme type interactions studied in reference 13, which cover a rather wide range of isospin properties, $a_{\mathrm{cs}}$ has been found large and positive (in the range $20 \ldots 120 \mathrm{MeV}$ ) so that including it would enhance the effect of the curvature energy.

Finally the Coulomb energy will be treated as in the standard LDM : diffuseness and exchange corrections are known to be shape-independent [6] and the Coulomb redistribution energy in the droplet model is significantly smaller than the curvature and compression energies [16]. As already mentioned, we want to investigate here a qualitative and large effect so that we keep only the leading terms beyond the LDM.

The set of parameters used in the following are the ones calculated for Gogny's force :

$$
\begin{aligned}
a_{\mathrm{s}} & =20.1 \mathrm{MeV}[14] \\
a_{\mathrm{ss}} & =-38.0 \mathrm{MeV}[11,14] \\
a_{\mathrm{Coul}} & =\frac{3 e^{2}}{5 r_{\mathrm{nm}}}=0.768 \mathrm{MeV} \\
K & =228 \mathrm{MeV},
\end{aligned}
$$

and we shall study the potential energy surface as a function of the curvature energy $a_{\mathrm{c}}$ which, as we stated above, lies in the range $0 \mathrm{MeV}(\mathrm{LDM})$ to about $10 \mathrm{MeV}$. For the Gogny force, $a_{\mathrm{c}}$ has not been calculated but we rely on equation 1 to predict that it has to be in the same range of values than found for the Skyrme forces studied in reference 10.

For the family of shapes underlying we use the symmetric Lawrence shapes in cylindrical coordinates $[16,18]$ and convert the elongation and constriction parameters $z_{0}, z_{2}$ by nonlinear transformations to

$$
\begin{aligned}
& Q_{2}=2 A \int \mathrm{d}^{3} r r^{2} P_{2}(\theta) / V \\
& Q_{4}=8 A \int \mathrm{d}^{3} r r^{4} P_{4}(\theta) / V
\end{aligned}
$$

where $V$ is the volume of the nucleus. 
Let us now proceed with the discussion of our results. A rough way of evaluating the mean slope of the fission valley is to calculate the difference $\Delta E=E_{\mathrm{sad}}-E_{\mathrm{scis}}$ between saddle and scission energies. The corresponding plots are shown in figure 1 . On the vertical axis $\left(a_{\mathrm{c}}=0\right)$ are also given the LDM points obtained when neglecting both curvature and compression energies. Note that the compression term is negative, so that for small curvature energies the saddle point energy is smaller than the LDM value ( $2.6 \mathrm{MeV}$ for $a_{\mathrm{c}}=0$ but with compression effect, instead of $6.5 \mathrm{MeV}$ ). The saddle point energy then increases with $a_{\mathrm{c}}$ and reaches the value 11.2 MeV for $a_{\mathrm{c}}=10 \mathrm{MeV}$.

The scission energy shows the same behaviour (indeed it is enhanced because the deformations are larger) : inclusion of the compression effect alone brings $E_{\text {scis }}$ down to $-51 \mathrm{MeV}$, compared to the LDM value of $-33 \mathrm{MeV}$; inclusion of a curvature energy of $10 \mathrm{MeV}$ then raises $E_{\text {scis }}$ up to $-3 \mathrm{MeV}$. The net result on $\Delta E$ can be seen on the right part of figure 2 . Around the value $a_{\mathrm{c}}=6 \mathrm{MeV}$ compression and curvature effects cancel approximately and one recovers the LDM value of $-38 \mathrm{MeV}$, so that it is only above this value that one may expect the potential energy landscape to really change. This is a very interesting result, because it shows that if the curvature energy were not larger, one would in fact be entitled to rely on the LDM, thus neglecting two effects which would almost compensate. Around $a_{\mathrm{c}}=10 \mathrm{MeV}$, however, $\Delta E$ is reduced by a factor of about 3 with respect to $\Delta E_{\mathrm{LDM}}$ !

This schematic analysis is confirmed by the results of the more detailed calculations presented in figure 2. Here we show the equipotential lines from saddle to exit corresponding to 4 different cases : the LDM case (no compression) and 3 values of $a_{\mathrm{c}}: 5,8$ and $10 \mathrm{MeV}$, including the compression energy. For $a_{\mathrm{c}}=5 \mathrm{MeV}$ one sees that the mean slope of the fission valley has not changed much from the LDM case. For $a_{\mathrm{c}}=8 \mathrm{MeV}$, the slope decreases while the fission valley gets narrower. For $a_{\mathrm{c}}=10 \mathrm{MeV}$, the difference in energy between saddle and exit is now $\sim 4 \mathrm{MeV}$ instead of $\sim 14 \mathrm{MeV}$ in the LDM and the width of the valley is still smaller - a feature which is as well in semi-quantitative agreement when comparing with BGG's result as is the overall shape of the landscape in figure $2 d$ [2]. In fact, the distance between saddle and exit is now larger than in the microscopic calculation so that the slope is even smaller than in BGG's paper. However, the different terms neglected in our simple approach may have a relatively large effect on a slope which has been reduced by a factor of $\sim 3$. In particular we have neglected the possible rôle of the constant term in the mass formula. The different components of this term as well as their shape dependence can be derived from the leptodermous expansion. One can show that they are all negative [11] and will tend to slightly reduce the effect of the curvature energy. Shell effects also will have a relatively larger importance on a flat surface than on a steep one. Nevertheless we think that none of the missing corrections can produce a change comparable to that of the curvature energy.

The general features discussed above are not typical of the Gogny force : one can start with different values of the coefficients of the mass formula and check that the same effect on the mean slope is obtained when adding curvature and compression energies. But of course the whole potential landscape may be shifted downward or upward in energy depending on the values of the coefficients. It is then interesting to discuss the value of the saddle point energy $E_{\text {sad }}$ obtained with the Gogny force, in relation with the corresponding mass formula coefficients. The microscopic HFB calculation gives $E_{\text {sad }} \simeq 17 \mathrm{MeV}$ for symmetric left-right configurations and $E_{\text {sad }} \simeq$ $13 \mathrm{MeV}$ when this constraint is released [19]. After correcting for zero-point energies, $E_{\mathrm{sad}}$ is brought down to $8 \mathrm{MeV}$, which is still too high compared to the experimental value $5 \mathrm{MeV}$. The value found in the present work $E_{\mathrm{sad}}=11.2 \mathrm{MeV}$ (for $a_{\mathrm{c}}=10 \mathrm{MeV}$ ) reflects this discrepancy. A possible explanation of this could of course be that the inclusion of further correlations brings the barrier down to the correct experimental value. However, one may also speculate whether a possible explanation cannot be found in the macroscopic properties of the Gogny force. First, notice that the value of the surface energy $a_{\mathrm{s}}=20.1 \mathrm{MeV}$ is rather high. It is compa- 
rable to the values used in the macroscopic approaches of references 6,7 , but in these latter cases the curvature energy is zero so that the surface energy is somehow renormalized. On the other hand, one would expect a smaller surface energy when curvature energy is present. Another and more subtle aspect which could explain the discrepancy in the barrier height is related to the isospin properties of the interaction. The comparison between experimental and calculated masses in different series of isotopes ( $\mathrm{Ti}, \mathrm{Ni}, \mathrm{Zr}$ ) seems to indicate that the surface symmetry energy $a_{\mathrm{ss}}$ might be (in absolute value) too small (see 2 nd of Ref. 17). The same conclusion can be drawn from studies of the Giant Dipole Resonance [20], which favour a value of the ratio of surface symmetry energy to volume symmetry energy of $2 \ldots 3$ instead of 1.3 in the case of the Gogny force. Increasing $\left|a_{\mathrm{ss}}\right|$ by e.g. $25 \mathrm{MeV}$ (and readjusting consequently the volume symmetry energy) would decrease the effective surface energy in ${ }^{240} \mathrm{Pu}$ by $\sim 1 \mathrm{MeV}$, which would be enough to bring the calculated fission barrier to the experimental one. The same kind of readjustment on $a_{\text {ss }}$ (it is indeed a small readjustment) has been made in the case of SkM interaction [21] which lead to the Modified SkM force [22]; this last interaction reproduces correctly the ${ }^{240} \mathrm{Pu}$ barrier height.

In conclusion we have shown in the present investigation that the inclusion of a curvature term in the LDM calculation can drastically change the slope of the potential energy surface from saddle to exit and scission. The effect of compression dominates for values of the curvature energy $a_{\mathrm{c}}$ up to 5-6 MeV, leading to a slight increase of the mean slope; the opposite is true for $a_{\mathrm{c}}>6 \mathrm{MeV}$. The values of $a_{\mathrm{c}}$ obtained in an EDF approach exhibit little dependence on the effective interaction used and lie around $10 \mathrm{MeV}$. Such a value reduces the slope by a factor of $\sim 3$, which gives a surface in semi-quantitative agreement with the completely microscopic calculations of $\mathrm{BGG}$ in ${ }^{240} \mathrm{Pu}$. Arguments are given that the too high fission barrier might be due to a too small surface symmetry energy. The present study calls for more detailed investigations concerning the liquid drop parameters to all orders, inclusion of shell corrections [3, 23] and dynamical calculations allowing for tunnelling through the ridge. This question has been brought up by recent fission experiments [24] at the high flux reactor in Grenoble (ILL). As a matter of fact the fission processes at very high kinetic energies seem to support the idea that no quasi particle excitations are present in the fragments and that they are born in'compact configurations. A small number of quasiparticle excitations during the descent from saddle to exit has also been found in a recent calculation by Nifenecker et al. [25]. These different aspects seem to corroborate the idea of a weak slope of the potential energy surface beyond the barrier towards scission, and it could imply that the fission process is a frictionless motion of collective variables in a superfluid system at least up to the point of the more or less fast neck rupture.

\section{Acknowledgments.}

J. F. Berger and D. Gogny are especially acknowledged for their constant and fruitful interest in this work. We are also thankful for numerous discussions with M. Asghar, F. Gönnenwein, C. Signarbieux, H. Nifenecker and C. Guet.

\section{References}

[1] Berger, J. F., Girod, M. and Gogny, D., J. Physique Lett. 42 (1981) L-509.

[2] Berger, J. F., Private Communications and to be published.

[3] Brack, M., Damgaard, J., Jensen, A. S., Pauli, H. C., Strutinsky, V. M., Wong, C. Y., Rev. Mod. Phys. 44 (1972) 320;

Asghar, M. and RAMAMURThy, V. S., ILL-Grenoble preprint 1982.

[4] Buørnholm, S., Lynn, J. E., Rev. Mod. Phys. 52 (1980) 725.

[5] Strutinsky, V. M., Nucl. Phys. A 95 (1967) $420 ; 122$ (1968) 1. 
[6] Myers, W. D., Swiatecki, W. J., Ann. Phys. 84 (1974) 186.

Strutinsky, V. M., J. Exp. Theor. Phys. 45 (1964) 1891.

[7] Krappe, H. J., Nix, J. R., Sierk, A. J., Phys. Rev. C 20 (1979) 992.

[8] Von Groote, H., Hilf, E., Nucl. Phys. A 129 (1969) 513.

[9] Negele, J. W., Vautherin, D., Phys. Rev. C 5 (1972) 1472.

[10] Krivine, H., Treiner, J., J. Math. Phys. 22 (1981) 2484.

[11] Treiner, J., Krivine, H., IPNO/Th 82-18, Orsay Preprint.

[12] Myers, W. D., Swiatecki, W. J., Ann. Phys. 55 (1969) 395.

[13] Chu, Y. H., Jennings, B. K., BraCK, M., Phys. Lett. 68B (1977) 407.

[14] Brack, M., Guet, C., HÅkansson, H. B., to be published.

[15] Farine, M., Cote, J., Pearson, J. M., Nucl. Phys. A 338 (1980) 86.

[16] Hasse, R. W., Ann. Phys. 68 (1971) 377.

[17] Gogny, D., Proc. Int. Conf. on Nuclear Physics, München 73, ed. J. de Boer and H. J. Mang (NorthHolland).

Decharge, J., Gogny, D., Phys. Rev. C 21 (1980) 1568.

[18] Lawrence, J. N. P., Phys. Rev. 139 (1965) B-1227.

[19] Berger, J. F., GIROD, M., in Physics and Chemistry of fission 1979, vol. 1 (IAEA, Vienna, 1980) p. 265.

[20] Krivine, H., Schmit, C., Treiner, J., Phys. Lett. 112B (1982) 281 ;

LipParini, E., Stringari, S., Phys. Lett. 112B (1982) 421.

[21] Krivine, H., Treiner, J., Bohigas, O., Nucl. Phys. A 336 (1980) 155.

[22] Bartel, J., Quentin, P., Brack, M., Guet, C., Hakansson, H. B., Nucl. Phys. A 386 (1982) 79.

[23] Lounis, L., Fellah, M., Faid, B., Athimene, H., International Conference on Nuclear Data for Science and Technology, Antwerp 1982.

[24] Signarbieux, C., Montoya, M., Ribrag, M., Mazur, C., Guet, C., Perrin, P., Maurel, N. M., J. Physique Lett. 42 (1981) L-437.

Armbruster, P., Quade, U., Rudolph, K., Clerc, H. G., Mutterer, M., Pannicke, J., Schmitt, C., Theobald, J. P., Engelhardt, W., Gönnenwein, F., Schrader, H., Proceedings of the 4th International Conference on Nuclei far from Stability Helsingor, 1981.

[25] Nifenecker, H., Mariolopoulos, G., Bocquet, J. P., Brissot, R., Hamelin, Ch., Crancon, J., RISTORI, Ch., Z. Phys. A 308 (1982) 39. 